\title{
Functions of DEAD box RNA helicases DDX5 and DDX17 in chromatin organization and transcriptional regulation
}

\author{
Guillaume Giraud ${ }^{\#}$, Sophie Terrone ${ }^{\#}$ \& Cyril F. Bourgeois \\ Laboratoire de Biologie et Modelisation de la Cellule, Universite de Lyon, CNRS UMR 5239, INSERM U1210, Ecole Normale Superieure \\ de Lyon, Universite Claude Bernard Lyon 1, F-69007 Lyon, France
}

RNA helicases DDX5 and DDX17 are multitasking proteins that regulate gene expression in different biological contexts through diverse activities. Special attention has long been paid to their function as coregulators of transcription factors, providing insight about their functional association with a number of chromatin modifiers and remodelers. However, to date, the variety of described mechanisms has made it difficult to understand precisely how these proteins work at the molecular level, and the contribution of their ATPase domain to these mechanisms remains unclear as well. In light of their association with long noncoding RNAs that are key epigenetic regulators, an emerging view is that DDX5 and DDX17 may act through modulating the activity of various ribonucleoprotein complexes that could ensure their targeting to specific chromatin loci. This review will comprehensively describe the current knowledge on these different mechanisms. We will also discuss the potential roles of DDX5 and DDX17 on the 3D chromatin organization and how these could impact gene expression at the transcriptional and post-transcriptional levels. [BMB Reports 2018; 51(12): 613-622]

\section{INTRODUCTION}

DDX5 (p68) and DDX17 (p72) belong to the large family of evolutionarily conserved DEAD box RNA helicases (1). Because of their multiple molecular activities, these two proteins have several partially redundant functions in the regulation of gene expression, particularly in cell differentiation. Some of their most described functions include their control of nuclear microRNA processing and pre-mRNA alternative splicing, which is consistent with their ability to remodel ribonucleoprotein complexes. DDX5 and DDX17 share a

${ }^{*}$ Corresponding author. Tel: +33-4-7272-8663; Fax: +33-4-72728674; E-mail: cyril.bourgeois@inserm.fr

${ }^{\#}$ These authors contributed equally to this work.

https://doi.org/10.5483/BMBRep.2018.51.12.234

Received 10 September 2018

Keywords: Chromatin, DDX17, DDX5, RNA helicase, Transcription highly homologous ATPase domain $(90 \%$ homology in human proteins), and ATP binding and hydrolysis are necessary to catalyze the unwinding of local RNA secondary structures, as well as for their RNA annealing activity (2-4).

DDX5 and DDX17 are also important coregulators of various transcription factors, and they interact with a number of chromatin-associated factors (5). This may seem to conflict with their enzymatic activity, and it remains uncertain whether their helicase activity is required for this particular function. In animals, it is not known whether these proteins have an affinity for double-stranded DNA or RNA-DNA hybrids, which could be relevant for chromatin and transcription-related functions. However, note that the ortholog of DDX5 in peas (Pisum sativum Psp68) exhibits bidirectional DNA helicase activity (6). In this review, we will first update the different mechanisms by which DDX5 and DDX17 coregulate transcription factors, as they have been reviewed elsewhere (5), then we will specifically highlight the contribution of long noncoding RNA (IncRNAs) to DDX5/DDX17-mediated functions, as they help elucidate how RNA helicases could work in the context of ribonucleoprotein complexes. Finally, we will replace these mechanisms in a tridimensional genomic context and propose that DDX5/DDX17 may regulate specific aspects of gene expression by locally modulating the spatial and dynamic gene organization.

\section{DDX5 AND DDX17 REGULATE THE TRANSCRIPTIONAL ACTIVITY OF SEVERAL TRANSCRIPTION FACTORS IN VARIOUS CELLULAR CONTEXTS}

DDX5 and DDX17 regulate transcription through their direct interactions with several transcription factors (e.g. ER $\alpha, \mathrm{P} 53)$ (Table 1). As this has been reviewed previously (5), we will mostly focus below on the biological consequences of these interactions in normal or pathological situations.

The presence of DDX5 or DDX17 within transcription factor complexes may partly explain how the aberrant expression of DDX5 and/or DDX17 contributes to pathologies such as cancer (7-12). In addition to its well-described interaction with $\mathrm{ER} \alpha$ and its involvement in breast cancer (13-15), DDX17 also interacts with SOX2 in a subset of ER $\alpha$-positive breast cancer

ISSN: 1976-670X (electronic edition)

Copyright (c) 2018 by the The Korean Society for Biochemistry and Molecular Biology

(c) This is an open-access article distributed under the terms of the Creative Commons Attribution Non-Commercial License (http://creativecommons.org/licenses/by-nc/4.0) which permits unrestricted non-commercial use, distribution, and reproduction in any medium, provided the original work is properly cited. 
DDX5 and DDX17 in transcription and chromatin regulation

Guillaume Giraud, et al.

Table 1. Protein and noncoding RNA partners of DDX5 and DDX17

\begin{tabular}{|c|c|c|c|c|c|}
\hline Transcription factor & Other protein partner & ncRNA & DDX partner & Function - biological context & Reference \\
\hline TRP53 & & & DDX5/DDX17 & Apoptosis & (5) \\
\hline VDR & & & DDX5 & n.d. & \\
\hline$E R \alpha$ & CBP, p300 & $S R A$ & DDX5/DDX17 & Cell proliferation & \\
\hline AR & & & DDX5/DDX17 & & \\
\hline NF-KB p50 & & & DDX5 & & (8) \\
\hline SMADs & СBP & & DDX5/DDX17 & & $(18,20,21)$ \\
\hline SOX2 & & & DDX17 & & (16) \\
\hline NOTCH1/RBP-J & p300, MAML1 & $S R A$ & DDX5 & & $(53,94)$ \\
\hline STAT3 & & & DDX5 & & (95) \\
\hline E2F1 & & & DDX5 & & (5) \\
\hline \multirow[t]{2}{*}{ Beta-Catenin } & & & DDX5 & & $(9,12)$ \\
\hline & & $m r h l$ & DDX5 & Cell differentiation & (59) \\
\hline MYOD & BRG1, p300 & $S R A$ & DDX5/DDX17 & Myoblast differentiation & $(21,22)$ \\
\hline RUNX2 & & & DDX5 & Osteoblast differentation & $(23)$ \\
\hline REST & EHMT2 & & DDX17 & Neuronal differentiation & (24) \\
\hline \multicolumn{6}{|l|}{ Chromatin modifiers } \\
\hline HDAC1 & & & DDX5/DDX17 & n.d. & $(26-28)$ \\
\hline PRC2 - TrxG & & $S R A$ & DDX5 & $\begin{array}{l}\text { Pluripotency - HBV infection - } \\
\text { Reprogramming inhibition }\end{array}$ & $(30,55)$ \\
\hline SU(VAR)3-9 & & & Rm62 & Heat shock response & (44) \\
\hline \multicolumn{6}{|l|}{ Insulators } \\
\hline CTCF / Cohesin & & $S R A$ & DDX5 & $\begin{array}{l}\text { Gene insulation - 3D genome } \\
\text { organization }\end{array}$ & $(69,96)$ \\
\hline \multirow[t]{3}{*}{ CP190 } & & RNAi & Rm62 & Gene insulation & (67) \\
\hline & & MeXis & DDX17 & Lipid Homeostasis & (62) \\
\hline & & LOC284454 & DDX5 & Cell Migration - Breast cancer & (63) \\
\hline
\end{tabular}

Only transcription factors and chromatin-associated factors are indicated.

cells that respond to this transcription factor (16). In these stem cell-like cells, DDX17 contributes to the activation of SOX2-responsive genes by stabilizing SOX2 binding to its target promoters. DDX17 depletion is associated with a decrease of tumor characteristics (e.g. colony formation), highlighting its importance in breast tumorigenesis (16). Both RNA helicases were identified as SOX2 binding proteins in glioblastoma cells, suggesting that DDX5 may also be involved in SOX2 transcriptional activity (17).

DDX5 may also contribute to cancer development by modulating various signaling pathways. For example, DDX5 interacts with $\beta$-catenin in non-small-cell lung cancer cells as well as colorectal cancer cells, and it also promotes its nuclear translocation, which is associated with the coactivation of Wnt-responsive genes such as MYC or CCND1 $(12,18)$. DDX5 and $\beta$-catenin are also involved together in the regulation of androgen receptor (AR) transcriptional activity in prostate cancer cells, where DDX5 promotes the recruitment of both transcription factors to AR target genes (9). Finally, the interaction between DDX5 and $\beta$-catenin contributes to the epithelial to mesenchymal transition (EMT), a process involved in the formation of metastases (19). Interestingly, some function of DDX5 (and DDX17) in EMT is also supported by its interaction with SMAD proteins, which are mediators of the EMT-inducing TGF- $\beta$ pathway. DDX5 has been shown to enhance SMAD3 transcriptional activity in response to TGF- $\beta$ (20), and more recently, our group has shown that DDX5 and DDX17 directly control the SMAD4-dependent expression of master EMT factors SNAI1 and SNAI2 upon TGF- $\beta$ treatment (21).

This involvement of DDX5 and DDX17 in EMT, an embryonic trans-differentiation process, illustrates their contributions toward controlling cell fate transitions and cell differentiation. Accordingly, murine $\operatorname{Dd} \times 5$ and $\operatorname{Dd} \times 17$ are essential for the early stages of myoblast or osteoblast differentiation through their interaction with master transcription factors Myod or Runx2, respectively (21-23). In both cases, Ddx5 is recruited to Myod and Runx2 responsive promoters, and it enhances their transcriptional activity. During myogenesis, one consequence is the induced expression of myogenic microRNAs, myogenic transcription factors (Myog or Mef2c), as well as muscle specific genes $(21,22)$. 
Recently, we have shown on a genome-wide scale that an interaction between an RNA helicase and a transcription factor can also lead to gene repression (24). DDX17 and DDX5 are necessary for repressing the expression of a large subset of neuronal genes in undifferentiated neuroblastoma cells, in cooperation with the REST transcription factor. The bindings of both REST and DDX17 to their target promoters are mutually dependent on each other, but the way in which RNA helicases stabilize REST complex binding at the chromatin remains unknown.

\section{DDX5 AND DDX17 INTERACT WITH CHROMATIN MODIFIERS AND REMODELERS}

As described above, the contributions of DDX 5 and DDX17 to physiological or cancer-related processes may be mediated largely by their coregulator activity of transcription factors. As we will see below, DDX5 and DDX17 function by modulating chromatin accessibility at different levels, in an active or repressive way.

First, DDX5 and DDX17 have often been reported to modulate the post-translational modifications of histones. Both factors regulate histone acetylation positively or negatively by interacting with histone acetyltransferases or deacetylases. DDX5 and DDX17 interact and synergize with acetyltransferases CBP (CREB-binding protein) and p300 to activate transcription, such as in the context of SMAD3-mediated transcriptional activation $(20,25)$. In contrast, the interaction between RNA helicases and histone deacetylase 1 (HDAC1) contributes in vitro to the DDX5/DDX17-dependent corepression of specific promoters (26). Interestingly, this interaction is reinforced by the sumoylation of DDX5 and DDX17, which stabilizes both proteins but differentially affects their activities as transcriptional coregulators, depending on the tested promoter or transcription factor $(27,28)$.

DDX5 and DDX17 also have effects on histone methylation. In undifferentiated neuronal precursors, DDX17 is recruited to some of the REST-responsive promoters along with the histone methyltransferase EHMT2, and it is required for the binding of EHMT2 to chromatin (24). EHMT2 catalyzes the mono and dimethylation of histone $\mathrm{H} 3$ lysine 9 (H3K9me1/2) and contributes to REST-mediated repression (29). Our results suggest that a DDX17-controlled deposition of H3K9me2 mark to REST-responsive promoters could contribute to the RESTand DDX17-mediated repression of neuronal genes (24). In hepatocytes, DDX5 also interacts with several subunits of the Polycomb-repressive complex 2 (PRC2), including the histone methyltransferase EZH2, which catalyzes the trimethylation of histone H3 lysine 27 (30). Upon Hepatitis B virus (HBV) infection, DDX5 helps stabilize another PRC2 subunit, SUZ12, and hence allows for PRC2-mediated gene repression, particularly at key pluripotency genes. However, whether DDX5 is directly recruited to PRC2 target genes is yet to be tested (30).
Second, DDX5 and DDX17 interact with the BRG1 chromatin remodeler. In muscle cells, DDX5 and DDX17 recruit BRG1 to MYOD target genes, increasing the chromatin accessibility for the transcription machinery, which helps coactivate MYOD-dependent transcription (22).

Finally, several reports have suggested that DDX5 may be involved in the control of DNA methylation and/or demethylation of $\mathrm{CpG}$ dinucleotides, as it interacts with DNA methyltransferase 3 proteins (DNMT3A and B) as well as with thymine DNA glycosylase (TDG) (31-34). The exact role of DDX5 in the control of these processes is unclear. DDX5 is recruited to chromatin along with both DNMT3A/B and TDG proteins at the beginning of each transcription cycle of an $E R \alpha$-responsive promoter, suggesting some function in the subsequent DNA demethylation of this promoter (34). Interestingly, TDG-mediated demethylation activity requires its association with CpG-rich ncRNAs that serve as guides for the enzyme (35). This raises the possibility that DDX5 and DDX17 could control this process by modulating RNA-DNA rearrangements. Importantly, the DDX5/DDX17-mediated modulation of local DNA demethylation would likely impact the binding of CTCF, another DDX5/DDX17-interacting factor that controls chromatin 3D architecture (see below).

All of these results reinforce the idea that these factors act at different levels for the fine epigenetic control of gene expression. The consequences of DDX5 and DDX17 activity on the chromatin environment and on transcription depend on the nature of the complex that is recruited along with their transcription factor partner.

\section{THE REGULATORY ACTIVITY OF DDX5 AND DDX17 IN TRANSCRIPTION IS CONSERVED THROUGHOUT EVOLUTION}

Dbp2, the DDX5 yeast ortholog, is associated to chromatin in Saccharomyces Cerevisiae and contributes to the fine-tuning control of the GAL gene cluster, which is required for galactose metabolism (36-38). In a glucose-rich environment, Dbp2 corepresses GAL genes with Cyc8 (38). In the absence of Dbp2 or upon a glucose-to-galactose switch, long antisense ncRNAs overlapping the repressed genes accumulate and form RNA-DNA hybrids (R-loops) that evict Cyc8 from the promoters and contribute to gene activation (36). Dbp2 may prevent the formation of these R-loops, either by promoting the assembly of ribonucleoprotein complexes onto the ncRNAs or by stabilizing them $(37,39)$. Genome-wide studies have suggested that Dbp2 and Cyc8 control many genes involved in sugar transport and energetic metabolism through a similar mechanism (40). This indicates that Dbp2, which is rapidly exported to the cytoplasm upon glucose deprivation (41), integrates nutritional signals in order to control energy homeostasis, a function that may be conserved in higher eukaryotes $(42,43)$.

In Drosophila melanogaster, several reports have also 
highlighted a transcriptional silencing activity of the DDX5 ortholog, Rm62. Upon heat shock, Rm62 is recruited to the hsp70 promoter, along with the histone methyltransferase SU(VAR)3-9. This allows the level of H3K9 methylation to increase in order to re-silence $h s p 70$ expression rapidly after heat shock (44). In line with this report, Rm62 promotes the clearance of transcripts from the chromatin, which allows for the deactivation of gene expression (45).

An intriguing possibility is that these repressive functions of DDX5 contribute to the formation of heterochromatin in connection with the RNA interference (RNAi) machinery. The RNAi pathway, which involves small RNA complexed with members of the Argonaute (AGO) protein family, controls mRNA translation and degradation in the cytoplasm, but it also has major nuclear functions to modify chromatin structure and control transcription, from yeast to mammals (46). Interestingly, DDX5 interacts with Ago2-containing complexes both in drosophila and in human cells, and Rm62/Ddx5 is required for RNAi in flies $(47,48)$. This suggests that DDX5 may be involved in the double-stranded RNA-mediated epigenetic control of gene expression, a fascinating question which should be further explored in future studies.

\section{DDX5 AND DDX17 FORM COMPLEXES WITH LONG NON-CODING RNAS}

Long noncoding RNAs (IncRNAs) are key epigenetic regulators of gene expression that employ a variety of mechanisms to enhance or silence promoter activity; these include recruiting and/or guiding chromatin modifiers or remodelers to specific regions, repressing specific factors by acting as decoys or via allosteric inhibition, or regulating the 3D organization of the genome $(49,50)$. As mentioned above, IncRNAs have also been found to regulate transcription in yeast, underlining the conservation of their function throughout evolution. DDX5 and DDX17 interact with various IncRNAs in several cell types, which impacts the chromatin state and the transcription of their target genes.

\section{$S R A$}

The most studied DDX5/DDX17-associated IncRNA is the steroid nuclear receptor activator RNA (SRA) that has been identified as a coactivator of several steroid nuclear receptors (51), although it also interacts with other transcription factors. $S R A$ plays critical roles in diverse physiological and pathological processes, such as myogenesis, adipogenesis and cancer. Notably, alternative splicing generates several SRA isoforms that are expressed differently depending on the biological context, as during myogenesis, and some SRA variants code for a protein (SRAP) that antagonizes the cotranscriptional function of the IncRNA (52). This bifaceted aspect of the function of SRA should be accounted for when studying this IncRNA.

As with DDX5 and DDX17, SRA interacts with ER $\alpha, A R$,
Notch1, and MyoD $(22,51,53) . S R A$ coactivates the Notch pathway with Ddx5 in mouse Beko cells through its interaction with both the Notch intracellular domain and the downstream transcription factor RBP-J, as well as by promoting p300 recruitment to Notch target genes (53). During myogenic differentiation of mouse $\mathrm{C} 2 \mathrm{C} 12$ cells, both RNA helicases and $S R A$ coactivate the transcription factor MyoD, and the joint overexpression of $S R A$ and $\mathrm{Ddx} 5$ stimulates the MyoDinduced conversion of mouse embryonic fibroblasts in skeletal muscle cells (22). Whether SRA contributes to the Ddx5/Ddx17dependent recruitment of $\mathrm{Brg} 1$ to MyoD-activated promoters is unknown, and the function of SRA in the coactivation process remains unclear as well.

Importantly, SRA interacts with several factors involved in transcriptional silencing (54). Co-immunoprecipitation experiments have indicated that $S R A$ associates with both TrxG and PRC2 complexes, which catalyze the trimethylation of histones $\mathrm{H} 3$ on lysine 4 (H3K4me3, active chromatin) and lysine 27 (H3K27me3, repressed chromatin), respectively (55). Members of a particular class of transcriptionally poised promoters carry both marks (bivalent promoters) and often correspond to the genes involved in differentiation. A significant fraction of SRA binding sites on chromatin carry the bivalent mark, suggesting that SRA could simultaneously guide both complexes (55). Remarkably, DDX5 facilitates the interaction of SRA only with the TrxG complex, and not with the PRC2 complex, perhaps depending on the capacity of DDX5 to alter the secondary structure of the IncRNA. Interestingly, in human pluripotent stem cells, the chromatin sites co-occupied by both SRA and DDX5 carry the H3K4me3 mark more frequently than those bound only by $S R A$, and DDX5 promotes SRA-mediated H3K4 methylation (55).

These results suggest that the SRA IncRNA can act as a multimodal scaffold for several complexes, and that it can be dynamically regulated by RNA helicases. Depending on the genomic context, SRA could contribute to the modification of histones in three different ways (mono or bivalent trimethylation) through TrxG and PRC2 activity, but in the presence of DDX5, the SRA-containing complex could switch to a more active form, thereby favouring the activation of target genes.

In the same study, the authors showed that $S R A$ is required for reprogramming induced pluripotent stem cells (iPSC) (55). One hypothesis is that the IncRNA could be involved in the deposition of bivalent histone modifications, a phenomenon that has been found to increase during cell reprogramming (56). In this context, DDX5 would counteract the production of iPSC by promoting the SRA-TrxG association and limiting the number of bivalent sites. Interestingly, DDX5 has recently been identified as a barrier to iPSC reprogramming $(57,58)$. The mechanism proposed in that study was different, but diverse strategies could be employed by DDX5 to disturb this very complex process. 


\section{Mrhl}

The meiotic recombination hot spot locus ( $m r h l)$ IncRNA is involved in the regulation of the Wnt signaling pathway in spermatogonial cells, where it interacts with DDX5 at specific chromatin loci in a manner linked to the regulation of the neighbouring genes $(59,60)$. Mrhl has also been shown to recruit corepressors to the negatively regulated Sox8 promoter (61), indicating that it could act as a guide for repressive complexes. As most mrhl-regulated chromatin loci are bound by the IncRNA in a DDX5-dependent manner (60), DDX5 may allow for the correct folding of the IncRNA through its unwinding activity, although this hypothesis was not demonstrated in that study. Another hypothesis is that the IncRNA may retain the RNA helicase in the nucleus. Consequently, the activation of the Wnt pathway induced by mrhl downregulation may result at least in part from the relocation of DDX5 to the cytoplasm and from the DDX5-dependent nuclear translocation of $\beta$-catenin, as has been previously shown $(19,59)$.

\section{MeXis}

MeXis (macrophage-expressed LXR-induced sequence) is a chromatin-associated liver $X$ receptor (LXR)-responsive IncRNA that regulates in vivo the expression of several LXR-responsive genes involved in cholesterol homeostasis, including the Abca1 gene (62). Mechanistically, MeXis helps open the chromatin at the Abca1 locus. Ddx17 has been identified as one protein partner of MeXis, and it is involved in the activation of the Abca1 gene through its MeXis-dependent recruitment to chromatin. This suggests that the complex MeXis/Ddx17 contributes to the activation of LXR-responsive genes (62).

Altogether, these examples highlight the essential role of IncRNAs in the transcriptional corepression or coactivation by DDX5 and DDX17. It is likely that many IncRNAs interact with these proteins, and these interactions likely differ depending on the cell type or biological context. For example, 73 new DDX5-interacting IncRNAs have been recently identified in HEK 293T cells, including the chromatinassociated IncRNA LOC284454, which has a global impact on gene expression (63). It will therefore be important to determine whether the DDX5- and/or DDX17-dependent regulation of other transcription factors involves IncRNAs, as many of these factors (or their coregulators) have known RNA partners, like REST (64) or SOX2 (65).

\section{THE REGULATORY FUNCTIONS OF DDX5 AND DDX17 IN A TRIDIMENSIONAL CHROMATIN CONTEXT}

Thus far, we have discussed the transcription-related functions of DDX5 and DDX17 from a fairly basic perspective, describing their recruitment and/or stabilization to the chromatin along with a variety of protein and RNA cofactors, and the resulting epigenetic modifications affecting gene expression. However, we must consider these mechanisms in the context of a highly complex and dynamically regulated tridimensional organization of the genes, and more generally, of the nucleus (66). Increasing evidence suggests that DDX5 and DDX17 may have roles in controlling the spatial organization of genes, with direct implications on gene expression.

\section{DDX5/DDX17 and the regulation of gene insulation}

This role of DDX5 was first described in Drosophila. Rm62 has been shown to interact with insulator proteins, and with CP190 in particular, in an RNA-dependent manner $(67,68)$. Phenotypic and immunofluorescence analyses have shown that Rm62 negatively affects the function of the gipsy insulator as well as the formation of insulator bodies (67). The authors proposed that CP190-containing higher-order chromatin loops form onto insulator sequences and are stabilized by RNAs that may originate from the RNAi pathway. The recruitment of Rm62 may promote the dissociation of these protein-RNA insulator complexes through direct contacts with CP190 and RNAs. This function of Rm62 may be dependent on the type of insulator complex, as the activity of the Fab-8 insulator was not affected by mutation of the Rm62 gene (68).

In mammals, the main insulation factor is the zinc-finger protein CTCF (CCCTC-binding factor), which is also regulated by DDX5 and DDX17 (69). Both RNA helicases interact with CTCF in an SRA-dependent manner, but CTCF binding at the IGF2/H19 imprinted insulator locus depends neither on DDX5 nor on SRA. In contrast, binding of the Cohesin complex, which often associates with CTCF (see also below), is reduced in the absences of DDX5 and SRA, and DDX5 binding reciprocally depends on CTCF, Cohesin, and SRA. These results suggest a mechanism in which CTCF is recruited on the insulator, where it combines with Cohesin and DDX5/SRA to insulate the IGF2 gene from the enhancer region on the maternal allele. Supporting this model, the depletion of any of these components decreases IGF2/H19 insulator function and increases chromatin looping between the enhancer and the gene (69).

While those two studies $(67,69)$ seem to contradict each other, this difference could reflect an evolutionary divergence of the insulation process between drosophila and mammals, or may be the consequence of differential interactions (Rm62 interacts with CP190 and DDX5 with CTCF). Alternatively, DDX5 may have opposite effects on 3D chromatin organization by reinforcing a subset of local contacts while dissociating others, with a global effect on chromatin higher-order organization.

\section{Possible consequences for the regulation of transcription and mRNA processing}

Beyond its function in gene insulation, CTCF globally regulates gene expression through its binding to thousands of sites, 
which often mark boundaries between genomic regions defined as compartments, topologically associating domains (TADs), or loop domains, on a descending scale from the higher-order 3D organization of chromosomes to local chromatin folding (70). Locally, DNA folding occurs when two DNA-bound CTCF molecules are brought into close spatial proximity through a loop. This process is also mediated by Cohesin, a ring-like multiprotein complex which co-occupies most genomic CTCF sites (70). Accordingly, the depletion of CTCF or Cohesin leads to a profound disorganization of the 3D genome (71-73).

In order to appreciate what the function of DDX5 and DDX17 may be in this context, we will hereafter focus on the regulation of alternative splicing, a largely cotranscriptional process that has multiple connections with chromatin organization (74), and which is regulated by both RNA helicases and by CTCF at the level of hundreds of exons (21, $75,76)$. As described by the kinetic model of splicing regulation, RNA Polymerase II (RNAPII) pausing is important for the inclusion of weak, otherwise skipped exons (74). CTCF and/or Cohesin control RNAPII pausing at different transcription steps, like at promoter-proximal early elongation checkpoints and in the control of termination (75, 77-79). CTCF was initially proposed to control exon inclusion by acting as a roadblock for elongating RNAPII at the exon 5 of the CD45 gene, a DNA methylation-sensitive effect $(75,80)$. However, a recent report showed that CTCF binding at alternative exons correlates both with DNA loops joining these exons to their cognate promoters and with exon inclusion at the RNA level (81). This is consistent with earlier observations showing that DNA regions corresponding to alternative exons that are enriched in $\mathrm{CTCF} /$ Cohesin binding sites are in close spatial proximity to promoters (82).

Recently, the loop extrusion model has been proposed as one mechanism through which Cohesin and CTCF organize genome folding. In this model, Cohesin entraps and extrudes a loop of DNA that expands until it reaches boundaries corresponding to CTCF sites, at which point extrusion pauses $(83,84)$. Transcription is one of the motors that can promote and orient Cohesin sliding along chromatin fibers (85). Therefore, considering transcription in a context of dynamic loop extrusion has important consequences for our understanding of the regulation of the transcriptional fidelity and cotranscriptional processing of the primary transcript.

We propose that the control of CTCF on splicing should be reconsidered so as to integrate the tridimensional folding of the gene that results from a dynamic loop extrusion process. By altering the kinetics of RNAPII on chromatin at the level of specific exons, this may impact the inclusion or skipping of these exons at the RNA level. As reviewed here, the multiple regulatory activities of DDX5 and DDX17 on epigenetic modifiers and chromatin-associated factors, particularly their effects on CTCF/Cohesin binding, strongly suggest that some of their target exons may be controlled as a result of
DDX5/DDX17-regulated modifications of the chromatin state and/or DNA looping.

The mechanism by which DDX5/DDX17 could locally influence the chromatin binding of architectural proteins may involve RNA molecules. CTCF has a high affinity for RNA, and it binds to several IncRNAs involved in dosage compensation and $X$ chromosome inactivation (86). CTCF binding to RNA modulates its binding to DNA, either in cis (86) or in trans (87). Cohesin binding to chromatin is also regulated by ncRNA, such as eRNA (88) or the IncRNA Xist, which inactivates $X$ chromosome (89). Xist has been proposed to act both as a Cohesin evictor to prevent the formation of a transcription-favourable chromatin conformation, and as a scaffold to recruit repressive complexes in order to promote $X$ inactivation. The presence of both DDX5 and DDX17 in the Xist interactome (89) raises the possibility that both RNA helicases could influence these events through their ability to remodel the Xist RNP complex. One could extend this model to other IncRNAs that may specifically regulate CTCF/Cohesin binding to other genomic regions, such as alternative exons.

It is clear that DDX5 and DDX17 can regulate alternative splicing through other mechanisms, via a direct effect on the local folding of their targeted transcripts or via the recruitment of RNA binding cofactors $(21,24,90,91)$, and our intention is not to argue that DDX5/DDX17-mediated splicing regulation relies only on their chromatin-related functions. However, this is a compelling question that should be addressed in the future.

\section{CONCLUSION}

As evoked elsewhere in this review, several important issues must be explored further in order to more precisely understand the mechanisms supporting DDX5 and DDX17 functions in chromatin-related gene regulation. This includes, for example, the influence of post-translational modifications, the possible links with the RNAi pathway, or the identification of genes specifically targeted by the 2 RNA helicases. This particular task is difficult to address due to the functional redundancy of the two factors and their cross-regulation, but genome-wide approaches should allow for this objective to be achieved in the near future. Finally, the exact role of their helicase activity also remains only partially addressed. If the RNA-related functions of DDX5 and DDX17, specifically their splicing regulation, require an active ATPase domain $(15,21,76,91$, 92), luciferase assays have shown that ATPase-dead DDX5 or DDX17 are as efficient as wild type proteins in their activities as transcriptional coregulators (5). However, such experiments have not ruled out the possible recruitment of endogenous RNA helicases to the tested promoters, which would influence the result. Indeed, yeast-two hybrid experiments have shown that both wild type and helicase-dead DDX5 and DDX17 mutants form homo and heterodimers (93), and that both proteins efficiently co-immunoprecipitate from mammalian 
extracts (unpublished results). Specific experiments are therefore required to definitively test whether or not ATPase-dead proteins are able to compensate for the absence of the endogenous protein.

\section{ACKNOWLEDGEMENTS}

We thank our colleagues Franck Mortreux and Didier Auboeuf for their comments and critical reading of the manuscript. This work was supported by grants from the "Agence Nationale pour la Recherche" [ANR-16-CE12-0009-01], "AFM-Téléthon", "Fondation ARC" and "Ligue contre le Cancer" (Comités du Rhône et de la Loire). G.G. and S.T. are supported respectively by ANR and by a doctoral fellowship from "AFM-Téléthon".

\section{CONFLICTS OF INTEREST}

The authors have no conflicting interests.

\section{REFERENCES}

1. Bourgeois CF, Mortreux F, Auboeuf D (2016) The multiple functions of RNA helicases as drivers and regulators of gene expression. Nat Rev Mol Cell Biol 17, 426-438

2. Hirling $H$, Scheffner $M$, Restle $T$, Stahl H (1989) RNA helicase activity associated with the human p68 protein. Nature 339, 562-564

3. Rossler OG, Straka A, Stahl H (2001) Rearrangement of structured RNA via branch migration structures catalysed by the highly related DEAD-box proteins p68 and p72. Nucleic Acids Res 29, 2088-2096

4. Huang Y and Liu ZR (2002) The ATPase, RNA unwinding, and RNA binding activities of recombinant p68 RNA helicase. J Biol Chem 277, 12810-12815

5. Fuller-Pace FV (2013) The DEAD box proteins DDX5 (p68) and DDX17 (p72): multi-tasking transcriptional regulators. Biochim Biophys Acta 1829, 756-763

6. Tuteja N, Tarique M, Banu MS, Ahmad M, Tuteja R (2014) Pisum sativum p68 DEAD-box protein is ATP-dependent RNA helicase and unique bipolar DNA helicase. Plant Mol Biol 85, 639-651

7. Causevic M, Hislop RG, Kernohan NM et al (2001) Overexpression and poly-ubiquitylation of the DEAD-box RNA helicase p68 in colorectal tumours. Oncogene 20, 7734-7743

8. Wang R, Jiao Z, Li R, Yue H, Chen L (2012) p68 RNA helicase promotes glioma cell proliferation in vitro and in vivo via direct regulation of NF-kappaB transcription factor p50. Neuro Oncol 14, 1116-1124

9. Clark EL, Hadjimichael C, Temperley R, Barnard A, Fuller-Pace FV, Robson CN (2013) p68/DdX5 supports beta-catenin \& RNAP II during androgen receptor mediated transcription in prostate cancer. PLoS One 8, e54150

10. Wang HZ, Gao X, Yang JJ, Liu ZR (2013) Interaction between p68 RNA helicase and $\mathrm{Ca} 2+$-calmodulin promotes cell migration and metastasis. Nature Communi- cations 4, 1354

11. Guturi KK, Sarkar M, Bhowmik A, Das N, Ghosh MK (2014) DEAD-box protein p68 is regulated by betacatenin/transcription factor 4 to maintain a positive feedback loop in control of breast cancer progression. Breast Cancer Research 16, 496

12. Wang Z, Luo Z, Zhou L, Li X, Jiang T, Fu E (2015) DDX5 promotes proliferation and tumorigenesis of non-smallcell lung cancer cells by activating beta-catenin signaling pathway. Cancer Sci 106, 1303-1312

13. Wortham NC, Ahamed E, Nicol SM et al (2009) The DEAD-box protein p72 regulates ER alpha-/oestrogendependent transcription and cell growth, and is associated with improved survival in ER alpha-positive breast cancer. Oncogene 28, 4053-4064

14. Dutertre M, Gratadou L, Dardenne E et al (2010) Estrogen regulation and physiopathologic significance of alternative promoters in breast cancer. Cancer Res 70, 3760-3770

15. Samaan S, Tranchevent LC, Dardenne E et al (2014) The Ddx5 and Ddx17 RNA helicases are cornerstones in the complex regulatory array of steroid hormone-signaling pathways. Nucleic Acids Res 42, 2197-2207

16. Alqahtani H, Gopal K, Gupta N et al (2016) DDX17 (P72), a Sox2 binding partner, promotes stem-like features conferred by Sox 2 in a small cell population in estrogen receptor-positive breast cancer. Cell Signal 28, 42-50

17. Fang X, Yoon JG, Li L et al (2011) Landscape of the SOX2 protein-protein interactome. Proteomics 11, 921-934

18. Yang LQ, Lin C, Zhao S, Wang H, Liu ZR (2007) Phosphorylation of p68 RNA helicase plays a role in platelet-derived growth factor-induced cell proliferation by up-regulating cyclin D1 and c-Myc expression. J Biol Chem 282, 16811-16819

19. Yang L, Lin C, Liu ZR (2006) P68 RNA helicase mediates PDGF-induced epithelial mesenchymal transition by displacing Axin from beta-catenin. Cell 127, 139-155

20. Warner DR, Bhattacherjee V, Yin X et al (2004) Functional interaction between Smad, CREB binding protein, and p68 RNA helicase. Biochem Biophys Res Commun 324, 70-76

21. Dardenne E, Polay Espinoza M, Fattet L et al (2014) RNA helicases DDX5 and DDX17 dynamically orchestrate transcription, miRNA, and splicing programs in cell differentiation. Cell Rep 7, 1900-1913

22. Caretti G, Schiltz RL, Dilworth FJ et al (2006) The RNA helicases p68/p72 and the noncoding RNA SRA are coregulators of MyoD and skeletal muscle differentiation. Dev Cell 11, 547-560

23. Jensen ED, Niu L, Caretti G et al (2008) p68 (Ddx5) interacts with Runx2 and regulates osteoblast differentiation. J Cell Biochem 103, 1438-1451

24. Lambert MP, Terrone S, Giraud G et al (2018) The RNA helicase DDX17 controls the transcriptional activity of REST and the expression of proneural microRNAs in neuronal differentiation. Nucleic Acids Res 46, 76867700

25. Rossow KL and Janknecht R (2003) Synergism between p68 RNA helicase and the transcriptional coactivators CBP and p300. Oncogene 22, 151-156

26. Wilson BJ, Bates GJ, Nicol SM, Gregory DJ, Perkins ND, 
Fuller-Pace FV (2004) The p68 and p72 DEAD box RNA helicases interact with HDAC1 and repress transcription in a promoter-specific manner. BMC Mol Biol 5, 11

27. Jacobs AM, Nicol SM, Hislop RG, Jaffray EG, Hay RT, Fuller-Pace FV (2007) SUMO modification of the DEAD box protein 068 modulates its transcriptional activity and promotes its interaction with HDAC1. Oncogene 26, 5866-5876

28. Mooney SM, Grande JP, Salisbury JL, Janknecht R (2010) Sumoylation of p68 and p72 RNA helicases affects protein stability and transactivation potential. Biochemistry 49, $1-10$

29. Kim HT, Jeong SG, Cho GW (2016) G9a inhibition promotes neuronal differentiation of human bone marrow mesenchymal stem cells through the transcriptional induction of RE-1 containing neuronal specific genes. Neurochem Int 96, 77-83

30. Zhang H, Xing Z, Mani SK et al (2016) RNA helicase DEAD box protein 5 regulates Polycomb repressive complex 2/Hox transcript antisense intergenic RNA function in hepatitis B virus infection and hepatocarcinogenesis. Hepatology 64, 1033-1048

31. Jost JP, Schwarz S, Hess D et al (1999) A chicken embryo protein related to the mammalian DEAD box protein $\mathrm{p} 68$ is tightly associated with the highly purified protein-RNA complex of 5-MeC-DNA glycosylase. Nucleic Acids Res 27, 3245-3252

32. Gallais R, Demay F, Barath P et al (2007) Deoxyribonucleic acid methyl transferases $3 a$ and $3 b$ associate with the nuclear orphan receptor COUP-TFI during gene activation. Mol Endocrinol 21, 2085-2098

33. Mpakali A, Kotini D and Agalioti T (2008) p68/Ddx5 RNA helicase is a key regulator of de novo DNA methyltransferases in mouse embryonic stem cells. FEBS J 275, 418 (PP8-125)

34. Metivier R, Gallais R, Tiffoche C et al (2008) Cyclical DNA methylation of a transcriptionally active promoter. Nature 452, 45-50

35. Jost JP, Frémont $M$, Siegmann M, Hofsteenge J (1997) The RNA moiety of chick embryo 5-methylcytosine- DNA glycosylase targets DNA demethylation. Nucleic Acids Res 25, 4545-4550

36. Cloutier SC, Wang S, Ma WK et al (2016) Regulated Formation of IncRNA-DNA Hybrids Enables Faster Transcriptional Induction and Environmental Adaptation. Mol Cell 61, 393-404

37. Cloutier SC, Ma WK, Nguyen LT, Tran EJ (2012) The DEAD-box RNA helicase Dbp2 connects RNA quality control with repression of aberrant transcription. J Biol Chem 287, 26155-26166

38. Cloutier SC, Wang S, Ma WK, Petell CJ, Tran EJ (2013) Long noncoding RNAs promote transcriptional poising of inducible genes. PLoS Biol 11, e1001715

39. Ma WK, Paudel BP, Xing Z, Sabath IG, Rueda D, Tran EJ (2016) Recruitment, Duplex Unwinding and ProteinMediated Inhibition of the Dead-Box RNA Helicase Dbp2 at Actively Transcribed Chromatin. J Mol Biol 428, 1091-1106

40. Wang S, Xing Z, Pascuzzi PE, Tran EJ (2017) Metabolic Adaptation to Nutrients Involves Coregulation of Gene
Expression by the RNA Helicase Dbp2 and the Cyc8 Corepressor in Saccharomyces cerevisiae. G3 (Bethesda) 7, 2235-2247

41. Beck ZT, Cloutier SC, Schipma MJ, Petell CJ, Ma WK Tran EJ (2014) Regulation of glucose-dependent gene expression by the RNA helicase Dbp2 in Saccharomyces cerevisiae. Genetics 198, 1001-1014

42. Xing Z, Wang S, Tran EJ (2017) Characterization of the mammalian DEAD-box protein DDX5 reveals functional conservation with S. cerevisiae ortholog Dbp2 in transcriptional control and glucose metabolism. RNA 23, 1125-1138

43. Mazurek A, Park Y, Miething C et al (2014) Acquired dependence of acute myeloid leukemia on the DEAD-box RNA helicase DDX5. Cell Rep 7, 1887-1899

44. Boeke J, Bag I, Ramaiah MJ et al (2011) The RNA helicase Rm62 cooperates with SU(VAR)3-9 to re-silence active transcription in Drosophila melanogaster. PLoS One 6, e20761

45. Buszczak M and Spradling AC (2006) The Drosophila P68 RNA helicase regulates transcriptional deactivation by promoting RNA release from chromatin. Genes Dev 20, 977-989

46. Kalantari R, Chiang CM, Corey DR (2016) Regulation of mammalian transcription and splicing by Nuclear RNAi. Nucleic Acids Res 44, 524-537

47. Ishizuka A, Siomi MC, Siomi H (2002) A Drosophila fragile $\mathrm{X}$ protein interacts with components of RNAi and ribosomal proteins. Genes Dev 16, 2497-2508

48. Hock J, Weinmann L, Ender C et al (2007) Proteomic and functional analysis of Argonaute-containing mRNA-protein complexes in human cells. EMBO Rep 8, 1052-1060

49. Mele M and Rinn JL (2016) "Cat's Cradling" the 3D Genome by the Act of LncRNA Transcription. Mol Cell 62, 657-664

50. Long Y, Wang X, Youmans DT, Cech TR (2017) How do IncRNAs regulate transcription? Sci Adv 3, eaao2110

51. Lanz RB, McKenna NJ, Onate SA (1999) A steroid receptor coactivator, SRA, functions as an RNA and is present in an SRC-1 complex. Cell 97, 17-27

52. Hube F, Velasco G, Rollin J, Furling D, Francastel C (2011) Steroid receptor RNA activator protein binds to and counteracts SRA RNA-mediated activation of MyoD and muscle differentiation. Nucleic Acids Res 39, 513-525

53. Jung C, Mittler G, Oswald F, Borggrefe T (2013) RNA helicase Ddx5 and the noncoding RNA SRA act as coactivators in the Notch signaling pathway. Biochim Biophys Acta Cell Res 1833, 1180-1189

54. Liu C, Wu HT, Zhu N et al (2016) Steroid receptor RNA activator: Biologic function and role in disease. Clin Chim Acta 459, 137-146

55. Wongtrakoongate $P$, Riddick $G$, Fucharoen $S$, Felsenfeld G (2015) Association of the Long Non-coding RNA Steroid Receptor RNA Activator (SRA) with TrxG and PRC2 Complexes. PLoS Genet 11, e1005615

56. Polo JM, Anderssen E, Walsh RM et al (2012) A Molecular Roadmap of Reprogramming Somatic Cells into iPS Cells. Cell 151, 1617-1632

57. Li H, Lai P, Jia J et al (2017) RNA Helicase DDX5 Inhibits 
Reprogramming to Pluripotency by miRNA-Based Repression of RYBP and its PRC1-Dependent and -Independent Functions. Cell Stem Cell 20, 462-477 e6

58. Bourgeois CF and Auboeuf D (2017) The RNA helicase DDX5 is a reprogramming roadblock. Stem Cell Investig 4, 79

59. Arun G, Akhade VS, Donakonda S, Rao MR (2012) mrhl RNA, a long noncoding RNA, negatively regulates $\mathrm{Wnt}$ signaling through its protein partner Ddx5/p68 in mouse spermatogonial cells. Mol Cell Biol 32, 3140-3152

60. Akhade VS, Arun G, Donakonda S, Rao MR (2014) Genome wide chromatin occupancy of mrhl RNA and its role in gene regulation in mouse spermatogonial cells. RNA Biol 11, 1262-1279

61. Kataruka S, Akhade VS, Kayyar B, Rao MRS (2017) Mrhl Long Noncoding RNA Mediates Meiotic Commitment of Mouse Spermatogonial Cells by Regulating Sox8 Expression. Mol Cell Biol 37

62. Sallam T, Jones M, Thomas BJ et al (2018) Transcriptional regulation of macrophage cholesterol efflux and atherogenesis by a long noncoding RNA. Nat Med 24, 304-312

63. Das M, Renganathan A, Dighe SN et al (2018) DDX5/p68 associated IncRNA LOC284454 is differentially expressed in human cancers and modulates gene expression. RNA Biol 15, 214-230

64. Tsai MC, Manor O, Wan Y et al (2010) Long noncoding RNA as modular scaffold of histone modification complexes. Science 329, 689-693

65. Bogu GK, Soh BS, Stanton LW (2013) The long noncoding RNA RMST interacts with SOX2 to regulate neurogenesis. Mol Cell 51, 349-359

66. Dekker J and Mirny L (2016) The 3D Genome as Moderator of Chromosomal Communication. Cell 164, $1110-1121$

67. Lei EP and Corces VG (2006) RNA interference machinery influences the nuclear organization of a chromatin insulator. Nat Genet 38, 936-941

68. Moshkovich N, Nisha P, Boyle PJ, Thompson BA, Dale RK, Lei EP (2011) RNAi-independent role for Argonaute2 in CTCF/CP190 chromatin insulator function. Genes Dev 25, 1686-1701

69. Yao H, K Brick K, Evrard Y, Xiao T, Camerini-Otero RD, Felsenfeld G (2010) Mediation of CTCF transcriptional insulation by DEAD-box RNA-binding protein p68 and steroid receptor RNA activator SRA. Genes Dev 24, 2543-2555

70. Merkenschlager M and Nora EP (2016) CTCF and Cohesin in Genome Folding and Transcriptional Gene Regulation. Annu Rev Genomics Hum Genet 17, 17-43

71. Nora EP, Goloborodko A, Valton AL et al (2017) Targeted Degradation of CTCF Decouples Local Insulation of Chromosome Domains from Genomic Compartmentalization. Cell 169, 930-944 e22

72. Rao SSP, Huang SC, Glenn St Hilaire B et al (2017) Cohesin Loss Eliminates All Loop Domains. Cell 171, 305-320 e24

73. Schwarzer W, Abdennur N, Goloborodko A et al (2017) Two independent modes of chromatin organization revealed by cohesin removal. Nature 551, 51-56
74. Naftelberg S, Schor IE, Ast G, Kornblihtt AR (2015) Regulation of Alternative Splicing Through Coupling with Transcription and Chromatin Structure. Ann Rev Biochem 84, 165-198

75. Shukla S, Kavak E, Gregory M et al (2011) CTCF-promoted RNA polymerase II pausing links DNA methylation to splicing. Nature 479, 74-79

76. Dardenne E, Pierredon S, Driouch K et al (2012) Splicing switch of an epigenetic regulator by RNA helicases promotes tumor-cell invasiveness. Nat Struct Mol Biol 19, 1139-1146

77. Gullerova M and Proudfoot NJ (2008) Cohesin complex promotes transcriptional termination between convergent genes in S. pombe. Cell 132, 983-995

78. Paredes SH, Melgar MF, Sethupathy P (2013) Promoterproximal CCCTC-factor binding is associated with an increase in the transcriptional pausing index. Bioinformatics 29, 1485-1487

79. Laitem C, Zaborowska J, Tellier M et al (2015) CTCF regulates NELF, DSIF and P-TEFb recruitment during transcription. Transcription 6, 79-90

80. Marina RJ, Sturgill D, Bailly MA et al (2016) TET-catalyzed oxidation of intragenic 5-methylcytosine regulates CTCFdependent alternative splicing. EMBO J 35, 335-355

81. Ruiz-Velasco M, Kumar M, Lai MC et al (2017) CTCFMediated Chromatin Loops between Promoter and Gene Body Regulate Alternative Splicing across Individuals. Cell Syst 5, 628-637 e6

82. Mercer TR, Edwards SL, Clark MB et al (2013) DNase I-hypersensitive exons colocalize with promoters and distal regulatory elements. Nat Genet 45, 852-859

83. Fudenberg G, Imakaev M, Lu C, Goloborodko A, Abdennur N, Mirny LA (2016) Formation of Chromosomal Domains by Loop Extrusion. Cell Rep 15, 2038-2049

84. Haarhuis JHI, van der Weide RH, Blomen VA et al (2017) The Cohesin Release Factor WAPL Restricts Chromatin Loop Extension. Cell 169, 693-707 e14

85. Busslinger GA, Stocsits RR, van der Lelij P et al (2017) Cohesin is positioned in mammalian genomes by transcription, CTCF and Wapl. Nature 544, 503-507

86. Kung JT, Kesner B, An JY et al (2015) Locus-specific targeting to the $\mathrm{X}$ chromosome revealed by the RNA interactome of CTCF. Mol Cell 57, 361-375

87. Sun S, Del Rosario BC, Szanto A, Ogawa Y, Jeon Y, Lee JT (2013) Jpx RNA activates Xist by evicting CTCF. Cell 153, 1537-1551

88. Tsai PF, Dell'Orso S, Rodriguez J et al (2018) A MuscleSpecific Enhancer RNA Mediates Cohesin Recruitment and Regulates Transcription In trans. Mol Cell 71, 129141 e8

89. Minajigi A, Froberg J, Wei C et al (2015) Chromosomes. A comprehensive Xist interactome reveals cohesin repulsion and an RNA-directed chromosome conformation. Science 349, aab2276

90. Camats M, Guil S, Kokolo M, Bach-Elias M (2008) P68 RNA helicase (DDX5) alters activity of cis- and trans-acting factors of the alternative splicing of $\mathrm{H}$-Ras. PLoS One 3, e2926

91. Kar A, Fushimi K, Zhou X et al (2011) RNA helicase p68 (DDX5) regulates tau exon 10 splicing by modulating a 
stem-loop structure at the 5' splice site. Mol Cell Biol 31, 1812-1821

92. Laurent FX, Sureau A, Klein AF et al (2012) New function for the RNA helicase p68/DDX5 as a modifier of MBNL1 activity on expanded CUG repeats. Nucleic Acids Res 40, 3159-3171

93. Ogilvie VC, Wilson BJ, Nicol SM et al (2003) The highly related DEAD box RNA helicases p68 and p72 exist as heterodimers in cells. Nucleic Acids Res 31, 1470-1480
94. Lin S, Tian L, Shen H et al (2013) DDX5 is a positive regulator of oncogenic NOTCH1 signaling in T cell acute lymphoblastic leukemia. Oncogene 32, 4845-4853

95. Sarkar M, Khare V and Ghosh MK (2017) The DEAD box protein p68: a novel coactivator of Stat3 in mediating oncogenesis. Oncogene 36, 3080-3093

96. Fujita $\mathrm{T}$ and Fujii $\mathrm{H}$ (2011) Direct identification of insulator components by insertional chromatin immunoprecipitation. PLoS One 6, e26109 